\title{
Unilateral Cytomegalovirus Retinitis in a Child With Acute Lymphoblastic Leukemia While on Maintenance Chemotherapy
}

\author{
Rahaf A. Mandura ${ }^{1}$, Karim Talat ${ }^{2}$, Wasil Jastaniah ${ }^{3}$ \\ 1. Department of Ophthalmology, King Abdulaziz University, Jeddah, SAU 2. Department of Ophthalmology - \\ Vitreoretinal Surgery, King Abdulaziz Medical City, Jeddah, SAU 3. Department of Pediatrics, Princess Noorah \\ Oncology Center, King Abdulaziz Medical City, Ministry of National Guard Health Affairs, Jeddah, SAU
}

Corresponding author: Rahaf A. Mandura,dr.mandura@gmail.com

\begin{abstract}
Cytomegalovirus retinitis (CMVR) commonly affects immunocompromised individuals, including acquired immunodeficiency syndrome (AIDS), post-organ transplant recipients and allogeneic stem cell transplant recipients. CMVR occurring in the acute lymphoblastic leukemia (ALL) maintenance phase of chemotherapy is rare and has been described in the literature as isolated case reports or case series. We report a case of unilateral CMVR in a pediatric patient during maintenance phase therapy for ALL. A 14-year-old boy known case of T-cell ALL with CNS2a status, was treated according to the Children's Oncology Group (COG) AALL0434 protocol. Induction therapy consisted of the standard high-risk four drugs, in addition to intrathecal methotrexate. At week 166 of maintenance therapy, the child presented with painless progressive loss of vision in the right eye for one week. The best-corrected visual acuity (BCVA) of the right eye was 6/36 and the left eye was $6 / 6$. Dilated fundus examination of the right eye showed multiple large yellow-white cloudy chorioretinal lesions with areas of intraretinal hemorrhages in the macula, and overlaying focal vitritis. Optical coherence tomography (OCT) of the right eye showed macular edema and mild subretinal fluid. Cytomegalovirus polymerase chain reaction of the blood was detected with high quantitative value. A diagnosis of CMVR was made and an induction doses of intravenous ganciclovir was followed by maintenance doses of oral valganciclovir. Our case suggests that pediatric patients with ALL in the maintenance phase are considered immunocompromised and that physicians should be aware of CMVR incidence in such group. Early diagnosis and prompt treatment are important to preserve vision and prevent future visual morbidity.
\end{abstract}

Review began 05/09/2021 Review ended 05/19/2021 Published 05/26/2021

\section{(๑) Copyright 2021}

Mandura et al. This is an open access article distributed under the terms of the Creative Commons Attribution License CC-BY 4.0., which permits unrestricted use, distribution, and reproduction in any medium, provided the original author and source are credited.
Categories: Ophthalmology, Pediatrics, Infectious Disease

Keywords: cytomegalovirus, cytomegalovirus retinitis, acute lymphoblastic leukemia, pediatric, all, hematology

\section{Introduction}

Cytomegalovirus (CMV) is a type 5 herpes virus causing an asymptomatic or minimally symptomatic illness in immunocompetent individuals [1]. Furthermore, it is considered a common opportunistic intraocular infection in acquired immune deficiency syndrome (AIDS) secondary to the depressed cell-mediated immunity when CD4 counts decline to less than 100/ $\mu \mathrm{L}$ [2]. It can also occur in patients who received a solid organ or hematopoietic stem cell transplant [1]. Cytomegalovirus retinitis (CMVR) due to CMV infection is very destructive and can cause full-thickness retinal inflammation, hemorrhage, and necrosis which can eventually result in serious complications such as retinal detachment and vision loss [3]. CMVR starts by affecting the vascular endothelial cells. Later, it affects the retinal pigment epithelium. After such manifestations occur, the virus' final cytopathic effect is subsequent retinal necrosis [4]. Acute lymphoblastic leukemia (ALL) patients undergoing maintenance chemotherapy rarely develop CMVR and are usually described as isolated case reports or case series in the literature [5-9]. We describe a case of unilateral CMVR in a pediatric patient during his maintenance phase therapy for ALL.

\section{Case Presentation}

A 14-year-old boy who is a known case of T-cell acute ALL with CNS2a status presented with painless progressive loss of vision in the right eye for one week. Regarding his ALL history, he initially presented with leukocytosis of $572 \times 10^{9} / \mathrm{L}$ and flow cytometry confirmed T-cell immunophenotype. He had no clinical symptoms or signs of neurological involvement and his vision was intact. However, he had evidence of blasts with low white blood cells on cyto-spin in his cerebrospinal fluid; thus, his central nervous system (CNS) involvement was categorized as CNS2a. Leukemia cytogenetics showed 46XY, del(6)(q21q23). He was treated according to the Children's Oncology Group (COG) AALL0434 protocol [10]. Induction therapy consisted of the high-risk four-drug induction and intrathecal therapy. In addition, two extra intrathecal methotrexate doses were given during induction due to his CNS2a status. End-of-induction minimal residual disease (MRD) showed 6\% bone marrow blasts and at the end-of-consolidation, his MRD was negative with $<0.01 \%$ blasts. After completing the intensive phase therapy, he was started on maintenance therapy, which consisted of 12-week cycles of monthly vincristine, dexamethasone pulse therapy, daily oral 6- 


\section{Cureus}

mercaptopurine and weekly oral methotrexate [10]. He tolerated therapy relatively well until week 166 of maintenance therapy when a painless progressive loss of vision in the right eye started. Prior ocular history was unremarkable with no previous ocular disease while his past medical history was significant for varicella-zoster reactivation at the left thoracic dermatome during week 154 of maintenance therapy and was treated with intravenous acyclovir for 10 days with complete resolution. On ocular exam, the bestcorrected visual acuity (BCVA) of the right eye was 6/36 and the left eye was 6/6. Intraocular pressure measured by tonopen was $17 \mathrm{mmHg}$ in the right eye and $15 \mathrm{mmHg}$ in the left eye. Anterior segment examination was normal and anterior chambers were quiet in both eyes without evidence of keratic precipitates or anterior uveitis. Dilated fundus examination of the right eye showed multiple large yellowwhite cloudy inflammatory chorioretinal lesions with granular appearance and active borders, the largest

lesion measuring around three disc diameters which was located in the macular area extending to the center of the fovea centering around the major retinal arterial vasculature in the posterior pole and surrounding the optic disc. It was associated with significant full-thickness retinal necrosis, intraretinal hemorrhages, perivascular sheathing (perivasculitis) along the arcades, cotton wool spots (soft exudates) and mild focal vitritis overlying the whitish active lesions. No associated retinal breaks or rhegmatogenous retinal detachment were noted (Figure 1). Optical coherence tomography (OCT) of the right eye showed the necrotizing retina appeared swollen as compared to the surrounding areas with the presence of macular edema, mild subretinal fluid accumulation, disturbance of foveal architecture, hyperreflective intraretinal deposits and clumps of hyperreflective material in the posterior vitreous consistent with vitreous cells and debris (Figure 2) while the left eye was normal.

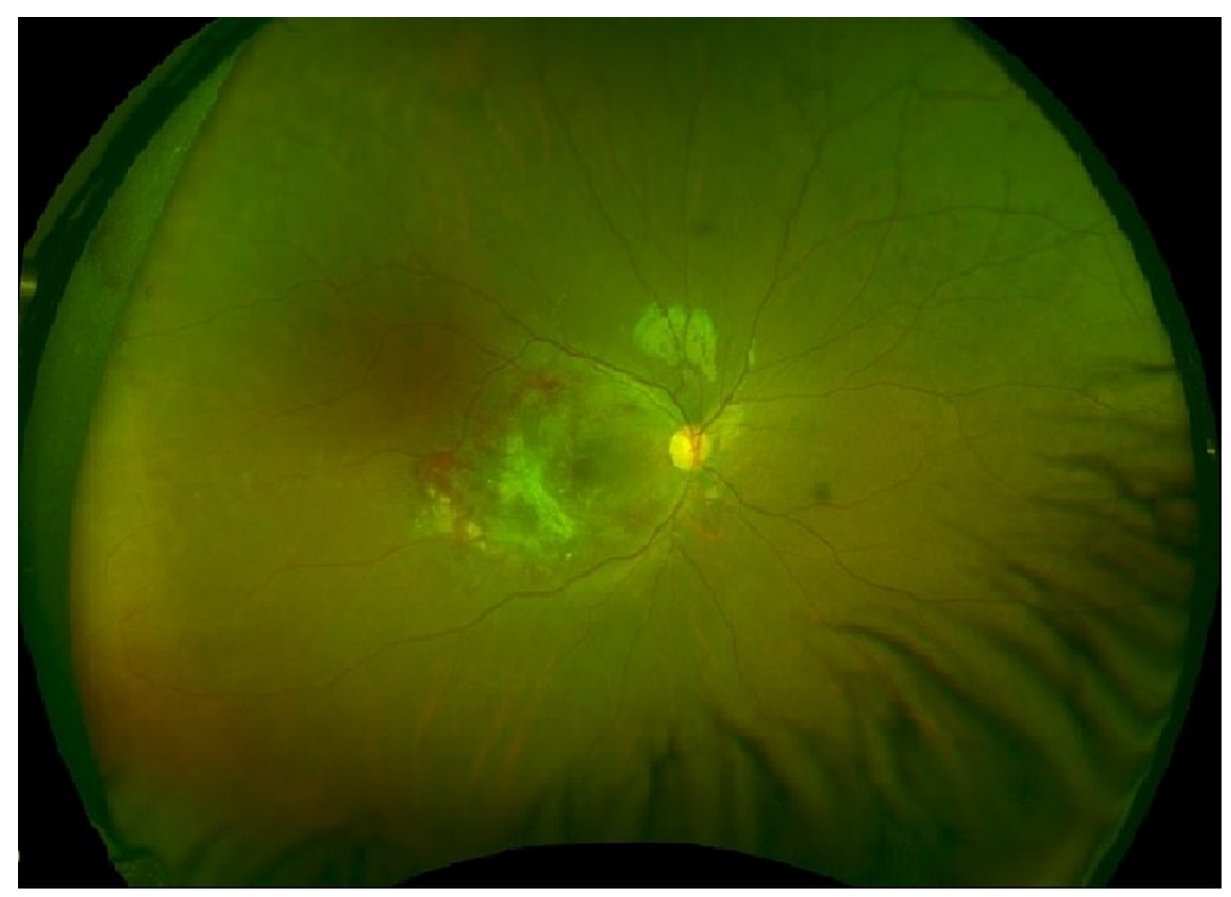

FIGURE 1: Fundus photo of the right eye showing CMV retinitis with retinal necrosis, intraretinal hemorrhages in the macula area, and focal overlying vitritis.

CMV: cytomegalovirus. 


\section{Cureus}

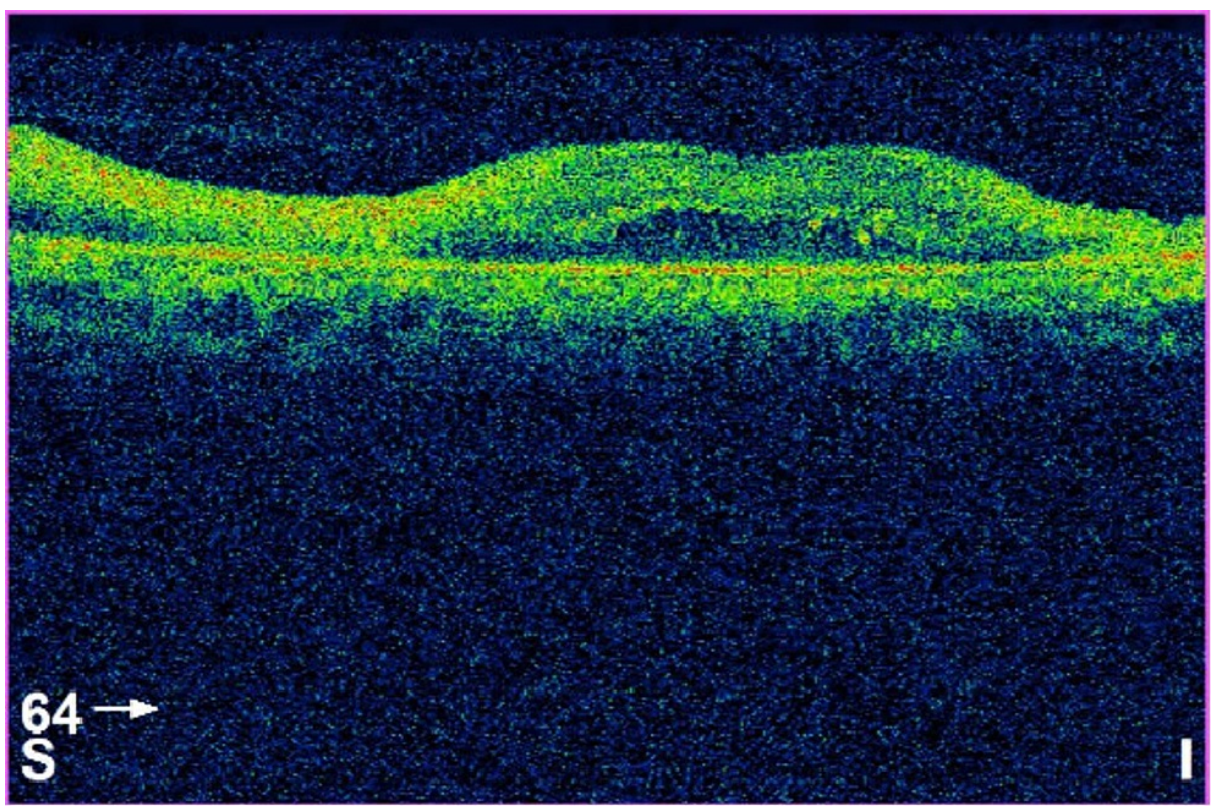

\section{FIGURE 2: Optical coherence tomography of the right eye showing macular edema and subretinal fluid.}

Blood test for CMV polymerase chain reaction (PCR) was performed, and CMV DNA was detected with the highest quantitative level of 409,000 copies $/ \mathrm{ml}$ and a CD 4 count of $2.5 \mathrm{cell} / \mathrm{mm}$. The patient was diagnosed with probable right eye CMVR with no evidence of any other organ involvement based on the clinical and laboratory findings. The maintenance chemotherapy was discontinued, and anti-CMV therapy was started in the form of high induction doses of daily intravenous infusion of ganciclovir $10 \mathrm{mg} / \mathrm{kg}$ for four weeks with weekly CMV PCR test. After the patient showed good response from the systemic therapy, no further intravitreal therapy was warranted. On examination, BCVA of the right eye improved to $6 / 30$, and the left eye was 6/6. Dilated fundus examination showed improvement with some resolution of the active retinitis and retinal hemorrhages. CMV PCR was repeated and showed a decreased quantitative level of 8,814 copies/ml.

On the second week's visit, BCVA of the right eye reached 6/9, and dilated fundus examination showed marked improvement and reduced size of retinitis lesions. Also, there was a considerable replacement of the retinal necrosis with thin whitish fibrous membrane and glial sheets and resolution of the focal vitritis with no sign of disease activity. CMV PCR dropped down to 622 copies/ml and OCT showed clear macula with complete resolution of subretinal fluid. On the subsequent days, the patient was kept on the maintenance doses of oral valganciclovir $900 \mathrm{mg}$ twice daily, which was continued for eight months until complete resolution of symptoms was achieved along with three consecutive negative readings of CMV PCR. The patient resumed and completed chemotherapy therapy a few months later and has been in clinical remission for two years.

\section{Discussion}

CMV viremia occurs in $13.6 \%$ of patients with lymphoid malignancies who did not receive stem cell transplantation [11]. CMVR is reported more frequently in children with AIDS than in other immunosuppressive conditions [12]. CMVR is characterized by spreading hematogenously to the retina, which happens after the systemic reactivation of latent infection [13]. An experienced ophthalmologist establishes the diagnosis of CMRV based on typical retina changes. Ocular pathophysiology of CMVR appears as full-thickness necrotizing retinitis. Furthermore, it appears as a fluffy, yellow-white retinal lesions, while intraretinal hemorrhage with little inflammation to the vitreous is an unpredictable sign found in the disease [14]. ALL patients developing CMVR happens mostly during maintenance chemotherapy. This maintenance phase is the most common period in the whole pediatric lymphoblastic group to develop CMV infection [15]. This finding could be due to the immunosuppressive chemotherapeutic agents used in this phase. It is hypothesized that the addition of dexamethasone and vincristine to methotrexate and 6-mercaptopurine may increase the risk of CMVR substantially [7]. The prevalence of CMV reactivation during the maintenance phase is often high and is caused by these drugs due to the development of lymphopenia [7].

We have treated our patient with intravenous ganciclovir for four weeks, followed by oral valganciclovir. The treatment was well tolerated by the patient without adverse effects and showed a good response resulting in a successful resolution of the CMVR, consistent with the literature [5-9]. The disadvantage of intravenous 
ganciclovir includes decreased bioavailability in ocular tissues in comparison to intravitreal ganciclovir and a significant relapse rate [12]. However, our patient was followed up for two years and no recurrence has been reported. In addition, systemic ganciclovir has the advantage of limiting the spread of CMV infection to the other eye and we believe that this prevention would not be possible if we considered intravitreal ganciclovir as a sole treatment.

\section{Conclusions}

CMVR is a tremendous visual threat in immunocompromised patients. Chemotherapy-related immunosuppression in pediatric patients with ALL happens in the maintenance phase of chemotherapy with chances of developing CMVR, making it important to have such a diagnosis in mind when seeing patients with ALL. A careful and vigilant approach to such cases is crucial as early diagnosis and prompt treatment are important to preserve vision and prevent visual morbidity.

\section{Additional Information \\ Disclosures}

Human subjects: Consent was obtained or waived by all participants in this study. King Abdulaziz Medical City issued approval NA. Both the patient and the guardian provided a written consent for the approval of publishing this case as long as the personal data stays anonymous. Conflicts of interest: In compliance with the ICMJE uniform disclosure form, all authors declare the following: Payment/services info: All authors have declared that no financial support was received from any organization for the submitted work. Financial relationships: All authors have declared that they have no financial relationships at present or within the previous three years with any organizations that might have an interest in the submitted work. Other relationships: All authors have declared that there are no other relationships or activities that could appear to have influenced the submitted work.

\section{References}

1. Gupta M, Shorman M: Cytomegalovirus. StatPearls, Treasure Island, FL; 2017.

2. Sudharshan S, Nair N, Curi A, Banker A, Kempen JH: Human immunodeficiency virus and intraocular inflammation in the era of highly active anti retroviral therapy - an update. Indian J Ophthalmol. 2020, 68:1787-98. 10.4103/ijo.IJO_1248_20

3. Munro M, Yadavalli T, Fonteh C, Arfeen S, Lobo-Chan AM: Cytomegalovirus retinitis in HIV and non-HIV individuals. Microorganisms. 2019, 8:10.3390/microorganisms8010055

4. Carmichael A: Cytomegalovirus and the eye. Eye. 2012, 26:237-40. 10.1038/eye.2011.327

5. Ahn B, Song S, Han MS, et al.: A case of cytomegalovirus retinitis during maintenance chemotherapy for acute leukemia. Pediatr Inf Vaccine. 2020, 27:198-204. 10.14776/piv.2020.27.e19

6. Dedania VS, Bhatnagar P, Santos RP, Kanwar VS: Bilateral cytomegalovirus retinitis in a child with acute lymphoblastic leukemia while on maintenance chemotherapy. Pediatr Hematol Oncol J. 2016, 1:35-7. 10.1016/j.phoj.2016.07.003

7. Moritake H, Kamimura S, Kojima H, et al.: Cytomegalovirus retinitis as an adverse immunological effect of pulses of vincristine and dexamethasone in maintenance therapy for childhood acute lymphoblastic leukemia. Pediatr Blood Cancer. 2013, 60:329-31. 10.1002/pbc.24298

8. Singh R, Singh R, Trehan A, Jain R, Bhalekar S: Cytomegalovirus retinitis in an ALL child on exclusive chemotherapy treated successfully with intravitreal ganciclovir alone. J Pediatr Hematol Oncol. 2013, 35:e118-9. 10.1097/MPH.0b013e31827078ad

9. Libby E, Movva S, Quintana D, Abdul-Jaleel M, Das A: Cytomegalovirus retinitis during chemotherapy with rituximab plus hyperfractionated cyclophosphamide, vincristine, doxorubicin, and dexamethasone. J Clin Oncol. 2010, 28:e661-2. 10.1200/JCO.2010.29.6467

10. Jastaniah W, Elimam N, Abdalla K, et al.: Early vs. late MRD response- and risk-based treatment intensification of childhood acute lymphoblastic leukemia: a prospective pilot study from Saudi Arabia. Exp Hematol Oncol. 2018, 7:29. 10.1186/s40164-018-0121-x

11. Styczynski J: Who is the patient at risk of CMV recurrence: a review of the current scientific evidence with a focus on hematopoietic cell transplantation. Infect Dis Ther. 2018, 7:1-16. 10.1007/s40121-017-0180-z

12. Celiker H, Karaaslan A, Kepenekli Kadayifci E, Atici S, Soysal A, Kazokoglu H, Koc A: Cytomegalovirus retinitis in an ALL child during maintenance therapy treated successfully with intravenous ganciclovir. Case Rep Ophthalmol Med. 2014, 2014:294238. 10.1155/2014/294238

13. Demir SÖ, Çeliker H, Karaaslan A, et al.: Cytomegalovirus retinitis in three pediatric cases with acute lymphoblastic leukemia: case series and review of the literature. Jpn J Infect Dis. 2016, 69:534-8. 10.7883/yoken.JJID.2015.223

14. Jain R, Trehan A, Mishra B, Singh R, Saud B, Bansal D: Cytomegalovirus disease in children with acute lymphoblastic leukemia. Pediatr Hematol Oncol. 2016, 33:239-47. 10.3109/08880018.2016.1173147

15. Thread: Cytomegalovirus (CMV) Retinitis: 36 year-old Indian male with HIV and decreasing visual acuity case. (2006). Accessed: May 2021: https://www.meduweb.com/showthread.php/27942-Cytomegalovirus(CMV)-Retinitis-36-year-old-Indian-male-with-HIV-and-dec.... 